\title{
Kernos
}

Revue internationale et pluridisciplinaire de religion grecque antique

16 | 2003

Varia

\section{Fr. LÉTOUBLON, Le mythe d'Orphée dans les métamorphoses d'Ovide}

\section{(2) OpenEdition \\ Journals}

Édition électronique

URL : http://journals.openedition.org/kernos/849

DOI : $10.4000 /$ kernos.849

ISSN : 2034-7871

\section{Éditeur}

Centre international d'étude de la religion grecque antique

\section{Édition imprimée}

Date de publication : 1 janvier 2003

Pagination : 377-379

ISSN : 0776-3824

\section{Référence électronique}

"Fr. LÉtoublon, Le mythe d'Orphée dans les métamorphoses d'Ovide », Kernos [En ligne], 16 | 2003, mis en ligne le 14 avril 2011, consulté le 22 septembre 2020. URL : http://journals.openedition.org/kernos/ 849 ; DOI : https://doi.org/10.4000/kernos.849 
précision le profil de ce rhéteur mégalomane et hypocondriaque dans le cadre contemporain du culte d'Asclépios et des doctrines sur l'interprétation des songes; l'A. souligne la conviction d'Aristide d'être un intermédiaire entre son dieu et les hommes, ainsi que la tendance hénothéiste que reflète le rapprochement Zeus-Asclépios qui se dégage des ses discours.

Le Destin et la Fortune sont le sujet d'étude du chapitre VI ( Daimon et Tyche nell'esperienza religiosa dell'uomo ellenistico : strutture ideologiche e pratiche cultuali », p. 255-301). Le concept de daimon, en singulier et en pluriel, est étudié par l'A. depuis Homère d'une façon remarquablement précise. Cette partie sert d'introduction à l'examen de la documentation concernant l'Agatbos Daimon, la Tyche et l'Agatbe Tyche, dans un effort pour nuancer et délimiter les traits de chaque culte, ainsi que de fixer le procédé d'entrecroisement de ces conceptions (avec une rigoureuse présentation des données épigraphiques par régions). Les deux derniers chapitres abordent deux aspects différents, mais complémentaires, du culte isiaque. D'un côté le rapprochement d'Isis et de la déesse Fortune (ch. VII, «Iside-Fortuna : Fatalismo e divinità sovrane del destino del mondo ellenistico-romano ", p. 303-325), ce qui mène l'A. non seulement à suivie les traces de la conjonction Isis-Fortuna (et en tant que fortuna videns), mais aussi de sa qualité de divinité cosmique. Les conclusions tirées des sources majoritairement littéraires (tout spécialement Apulée) sont confrontés à d'autres témoignages qui confirment l'accord avec la religiosité populaire. D'un autre côté, ce sont les aspects guérisseurs et de salut de la déesse qui composent le sujet d'étude de l'A. (ch. VIII, Iside Salutaris : aspetti medicali e oracolari del culto isiaco tra radici egiziane e metamorfosi ellenica ", p.327-342), qui souligne l'accord entre la tradition égyptienne sur les pouvoirs médicaux d'Isis et les pratiques magiques avec les courants religieux grecs de cette période.

Le résumé du contenu que je viens de faire ne donne qu'une idée très faible des vertus de cet ouvrage. Douée d'une remarquable formation historico-religieuse, avec une maîtrise incontestable dans la recherche des sources les plus variées, l'A. nous livre un très beau recueil. Ce n'est point une simple addition d'articles plus ou moins proches dans leur thématique, mais un ensemble bien atticulé qui forme une monographie indispensable pour comprendre la religiosité des premiers siècles de notre ère en général et pour la thématique oraculaire et du salut en particulier.

Emilio Suárez de la Torre (Universidad de Valladolid)

Françoise LÉToublon (coordonné par), Le mythe d'Orphée dans les métamorphoses d'Ovide, Paris, Adapt Éditions, 2001. 1 vol. $15 \times 21 \mathrm{~cm}, 128 \mathrm{p}$.

La première moitié de l'ouvrage, intitulće Orphée et le mythe de la poésie, est l'œuvre de la coordinatrice, Françoise Létoublon. Elle est divisée en quatre parties : 1 . Le personnage d'Orphée: un mythe sans substance? 2. Orphée avant Ovide. 3. Orphée dans la composition des Métamorphoses. 4. Les thèmes centraux du mythe d'Orphée. Elle s'achève par une bibliographie de 32 ouvrages : les articles ont été écartés car les A. cherchent moins à faire œuvre d'érudition qu'à ouvrir des pistes de recherche et s'adressent à un public plus étendu que celui des antiquisants (mais ceux-ci trouveront aussi de quoi nourrir leur réflexion dans ce petit ouvrage). L'A. note que le mythe d'Orphée est un mythe grec sans textes grecs des époques archäque et classique en dehors de fragments et d'allusions. On ne peut que regretter la disparition de la tragédie d'Eschyle intitulée les Bassarai, dont il reste vingt-neuf mots en quatre fragments et qui, semble-t-il, racontait ou représentait la mort d'Orphée. Les textes complets sont tous postérieurs à 200 ap. J.-C. Il s'agit d'une collection d'Hymnes, d'une épopée intitulée Argonautiques orphiques et d'un recueil poétique sur la puissance magique des pierres appelé Litbika. En revanche, les représentations figurées et les inscriptions sont abondantes et commencent dès le $\mathrm{vl}^{\mathrm{e}}$ siècle av. J.-C. De plus des découvertes archéologiques en provenance de tombes viennent enrichir le corpus: papyrus de Derveni sur des mystères orphiques, lamelles d'or ou plaques en os donnant des instructions pour le voyage dans l'au-delà. Le problème qui se pose aux chercheurs est qu'il est souvent difficile de faire le départ entre les doctrines dionysiaques, pythagoriciennes ou orphiques, tantôt proches, tantôt antagonistes. 
La première œuvre entièrement conservée où Orphée joue un rôle important est l'épopée d'Apollonios de Rhodes, les Argonautiques. Ce n'est pas un héros guerrier, mais un poète chanteur capable de charmer non seulement les hommes, mais aussi la nature inanimée, chênes sauvages ou rochers et jouant, à l'occasion, un rôle essentiel, ainsi quand, grâce à la vigueur de sa musique et de son chant, il sauve les Argonautes des Sirènes. On le retrouve au livre IV des Géorgiques de Virgile, dans un rôle mineur, à l'intérieur de l'épisode de l'invention de l'apiculture par Aristée. Celui-ci provoque indirectement la mort d'Eurydice : il la poursuit et elle est piquée par un serpent. Virgile se livre à un jeu intertextuel où l'on reconnaît des traces d'Homère, Hésiode et Apollonios. Mais c'est dans les livres X et XI des Métamorphoses d'Ovide qu'Orphée joue le rôle le plus important. En effet, on y trouve un subtil jeu d'enchâssement : Virgile raconte des métamorphoses; à l'intérieur de son récit, Orphée raconte des métamorphoses et, à l'intérieur du récit d'Orphée, Vénus raconte des métamorphoses. Orphée y apparaît comme le miroir du poète Ovide, comme dans les Géorgiques Protée apparaît comme le miroir de Virgile.

La clernière partie recense les thèmes centraux du mythe d'Orphée : l'amour et la mort (l'amour, comme la vie des hommes, est éphémère et fragile et l'histoire d'Adonis est le miroir de celle d'Orphée et d'Eurydice); la nature, la végétation et les métamorphoses; la chasse et l'amour, aux relations ambiguës; Pygmalion le sculpteur et la rivalité entre les arts : on découvre des ressemblances troublantes entre Hésiode (création de Pandora par Héphaïstos) et Ovide (la statue sculptée par Pygmalion à laquelle Vénus donne vie). L'étude de Fr. Létoublon s'achève par une suggestion originale : exilé à Olbia, ayant perdu le contact avec la société humaine, Ovide se compare à « une herbe inutile " (Pontiques, II, 1, 15), retrouvant ainsi la métamorphose en végétal, qui constitue l'un des thèmes les plus féconds des Métamorphoses.

Vianella Guyot intitule son étude des vers 1-29 du livre X des Métamorphoses «la quête d'Eurydice ». Elle souligne les différences de traitement du mythe par rapport à Virgile. Ovide s'attache à développer des thèmes non traités par son prédecesseur. La méthode n'est pas la même : Virgile est narratif, Ovide met en scène le personnage d'Orphée, dont il fait le substitut du poète et à qui, par un effet de mise en abyme, il fait raconter sept métamorphoses. Le monde supérieur est caractérisé par le mouvement et l'inquiétude, le monde inférieur par la fixité et l'effroi. Et l'homme, quant à lui, éprouve l'angoisse devant le mystère de la vie et de la mort. Mais ce n'est pas le cas du poète : du ton humble du début, Orphée passe à la revendication, au persiflage et au défi.

Albert Noguès s'intéresse aux vers 17 à 39 du livre X. Il voit dans le premier chant d'Orphée une distance ironique d'Ovide à l'égard de son héros et du sujet apparent du récit, parodie, jeu burlesque, désacralisation des dieux. Le ton épique ne doit pas être pris au sérieux, l'héroïsme est tourné en dérision et l'ironie du poète vise à la fois le poète et le lecteur.

Une autre étude de Vianella Guyot examine tout d'abord les « heurs et malheurs de l'œuvre à travers les siècles », en s'appuyant particulièrement sur l'ouvrage de G. Tronchet (La métamorphose à l'œuvre, Louvain/Paris, Peeters, 1998). L'engouement public ne s'est jamais démenti, Ovide faisant figure de passeur entre les mondes païen, chrétien et moderne. Mais, du vivant même d'Ovide, les clercs ont porté un regard critique, voire dédaigneux, parce que réducteur: on a vu dans les Métamorpboses un assemblage de compilations. Mais depuis 1980 on assiste à une réhabilitation. Les critiques soulignent l'unité et la spécificité des Métamorphoses, ouvrage fondé sur une esthétique en rupture totale avec les dogmes classiques augustéens. Loin d'être disparate, l'ouvrage est organisé selon une véritable stratégie narrative. On y relève l'extrême variété du cadre spatial et social avec les cloisons étanches entre le monde des hommes (activités de plein air, sport) et celui des femmes (activités familiales ou religieuses). Une constante : le thème de l'amour, présenté sous trois formes, pédérastie, inceste, amour conjugal. Quelques étourderies dans cet article utile: p. 93 écrire Taladoire et non "Taladoine, p. 95 rhétoriqueurs et non *'éthoriqueurs, Queneau et non "Quenault.

La demière étude, déjà publiée en 1999 dans la Revue de littérature comparée, est l'œuvre de Françoise Letoublon et Caroline Eades. Elle examine «le regard d'Orphée » dans les films de Théo Angelopoulos, et notamment dans le Regard d'Ulysse où l'on retrouve les trois mouvements qui jalonnent les récits consacrés à Orphée : la gloire, la 
chute, l'apothéose. L'article est suivi par la liste des films ayant Orphée comme personnage principal ou secondaire.

L'ouvrage se termine sur trois traductions des vers 14 à 41 du livre $X$ : Pierre du Ryer (1660), l'abbé Banier (1732), Georges Lafaye (1926). On notera avec amusement que la traduction de Pierre du Ryer fait sept lignes de plus que ses successeurs. C'est ce qu'on appelle « une belle infidèle». Un ouvrage utile, qui constitue un bon moyen d'approcher une oeuvre à laquelle les travaux récents, comme celui de G. Tronchet, ont enfin rendu justice.

Alain Moreau

(Université de Montpellier)

Anne-France Morand, Études sur les Hymnes orphiques, Leiden, Brill, 2001. 1 vol. $16 \times 24,5 \mathrm{~cm}, \mathrm{xI}+374 \mathrm{p}$. (Religions in the Graeco-Roman World, 143). ISBN : 90-04$12030-0$.

Cette étude commence par la reproduction du texte grec des Hymnes orphiques dans l'édition de Quandt (Berlin, 1955) avec quelques leçons différentes adoptées par l'A. et justifiées en notes. Après une brève introduction qui présente ces quatre-vingt-sept hymnes et annonce le but ambitieux de l'ouvrage, à savoir une relecture de ces textes à la lumière de nouveaux documents, tels les feuilles d'or et le papyrus de Derveni, et, partant, une redéfinition de l'orphisme à partir de l'ensemble des documents mis sous le nom d'Orphée, s'ouvre le premier chapitre d'un ouvrage qui en compte cinq. Ce chapitre traite de la question du genre des hymnes et, adoptant la division en "invocation », « développement » et « demande » proposée par Rudhardt, en examine les différentes parties pour aborder ensuite une étude globale du corpus envisagé cette fois dans son ensemble comme un tout cohérent ordonné logiquement. Ainsi, si chaque hymne peut être envisagé isolément, l'ensemble du traité n'en atteste pas moins des conceptions religieuses cohérentes. Au terme de cette étude formelle, une brève conclusion reste plus descriptive que significative malgré l'affirmation de l'A. qui dit s'être démarquée des autres historiens de la religion grecque. Le deuxième chapitre aborde le contenu des Hymmes avec la question des offrandes contenues dans leurs titres. Cette question en entraîne d'autres, sur le rapport entre l'offrande et le dieu qui la reçoit, les autres rituels décrits dans les textes et la communauté qui les pratiquait. Les conclusions sur ces questions restent floues et suggèrent à l'A. l'existence d'une communauté religieuse réelle, encore imprécisée à cet endroit de l'ouvrage. Le chapitre III veut cerner les croyances relatives aux dieux contenues dans ces textes afin de déterminer si elles correspondent à celles d'un groupe orphique. Les mises en rapport avec les cuvres littéraires et les inscriptions témoignent d'un ancrage dans la tradition assorti d'innovations propres aux textes attribués à Orphée. Quant à la date et au lieu d'origine de ces Hymnes, là encore, malgré le peu d'éléments permettant de les préciser - les références à Éleusis et à Dionysos qui y sont contenues ne sont pas un indice suffisant -, l'A. propose, sur base de témoignages épigraphiques, les premiers siècles de notre ère et une ville sans doute en rapport avec la mer d'Asie Mineure, privilégiant l'origine pergaménienne. Le quatrième chapitre traite de la présence dans les Hymnes de croyances en l'au-delà qui rapporteraient ces textes à une communauté orphique. Les premières conclusions de l'A. restent, de son propre aveu, très hypothétiques : la géographie des Enfers présentée dans les Hymnes ne diffère guère de la tradition, les passages sur une vie après la mott ainsi que les demandes liées à l'au-delà sont rares et il n'y a pas de référence explicite au meurtre de Dionysos par les Titans; aussi l'A. entreprend-elle une comparaison avec les lamelles d'or, tout en reconnaissant entre ces deux types de documents un grand écart de date et une différence de fonction. Les similitudes relevées ne semblent pas toujours significatives, comme la nécessité d'être pur. Le peu de références à l'au-delà dans les Hymnes par rapport aux lamelles est expliqué par l'A. par une différence dans la diffusion, supposée plus large dans le cas des Hymnes. Le chapitre $V$ envisage la question du groupe qui eut recours à ces Hymnes : s'agit-il d'un thiase ? Comment y sont appelés ses membres ? Il ressort d'un développement serré et fondé sur une abondance de témoignages épigraphiques que les termes utilisés, comme celui de mystes, ne sont pas spécifiques à ce genre de textes et n'ont donc pas été inventés dans le cadre de ceux-ci. Bref, au terme d'un ouvrage certes intéressant mais quelque peu 